\title{
In-situ observations of flux ropes formed in association with a pair of spiral
}

\section{nulls in magnetotail plasmas}

Ruilong Guo ${ }^{1}$, Zuyin $\mathrm{Pu}^{1,6}$, Li-Jen Chen ${ }^{2}$, Suiyan $\mathrm{Fu}^{1,6}$, Lun $\mathrm{Xie}^{1}$, Xiaogang Wang $^{3}$, Malcolm Dunlop ${ }^{4,5}$, Yulia V. Bogdanova ${ }^{5}$, Zhonghua Yao $^{7}$, Chijie Xiao ${ }^{8}$, Jiansen $\mathrm{He}^{1}$, Andrew N. Fazakerley ${ }^{7}$

${ }^{1}$ School of Earth and Space Sciences, Peking University, Beijing, 100871, China

${ }^{2}$ NASA Goddard Space Flight Center, Greenbelt, MD 20771, USA

${ }^{3}$ Harbin Institute of Technology, Harbin, 150001, China

${ }^{4}$ School of Astronautics, Beihang University, Beijing, 100191, China

${ }^{5}$ RAL Space, Rutherford Appleton Laboratory, STFC, Didcot, OX11 OQX, UK

${ }^{6}$ PKU/UCLA Joint Research Institute in Science and Engineering, Peking University, Beijing, China ${ }^{7}$ UCL Mullard Space Science Laboratory, Dorking, RH5 6NT, UK

${ }^{8}$ School of Physics, Peking University, Beijing, 100871, China

Signatures of secondary islands are frequently observed in the magnetic reconnection regions of magnetotail plasmas. In this paper, magnetic structures with the secondary-island signatures observed by Cluster are reassembled by a fitting-reconstruction method. The results show that three-dimensionally a secondary island event can manifest the flux rope formed with an $A_{s}$-type null and a $B_{s}$-type null paired via their spines. We call this $A_{s}$-spine- $B_{s}$-like configuration the helically wrapped spine model. The reconstructed field lines wrap around the spine to form the flux rope, and an $O$-type topology is therefore seen on the plane perpendicular to the spine. Magnetized electrons are found to rotate on and cross the fan surface, suggesting that both the torsional-spine and the spine-fan reconnection take place in the configuration. Furthermore, detailed analysis implies that the spiral nulls and flux ropes were locally generated nearby the spacecraft in the reconnection outflow region, indicating that secondary reconnection may occur in the exhaust away from the primary reconnection site.

\section{Introduction}

Magnetic reconnection is a process in plasma converting energy from the magnetic field to plasmas and changing magnetic topology for fast transportation of energy and particles. Existence of magnetic nulls is thought to be a critical element in three-dimensional (3D) 
reconnection for field-line breaking and reconnecting. Since successful operation of Cluster constellation, such nulls have been detected frequently in the magnetospheric and magnetosheath plasmas ${ }^{1-6}$. Field lines near a 3D null point are composed of two distinct families: the spine line $(\gamma$-line $)$ and the fan surface $(\Sigma \text {-surface })^{7,8}$. Clearly, if there is an electric current flowing along the spine line, the field lines in vicinity of the null will rotate around the spine line. It is then called a spiral null, but a radial null otherwise. For different directions of the field line on the fan surface with respect to the null, the nulls are further classified into two types of polarities: the negative $\left(A / A_{\mathrm{s}}\right.$-type, the subscript $s$ represents the spiral feature) and the positive ( $B / B_{\mathrm{s}}$-type). Various reconnection models with respect to a single null geometry have been proposed as the torsional-spine, the torsional-fan, or the spine-fan reconnection. ${ }^{8,9}$

In 3D geometry, both the two-dimensional (2D) $X$-point and $O$-point become neutral lines on which the magnetic field vanishes. Nevertheless, such a neutral line is structurally unstable, i.e., even an infinitesimal perturbation would break it into null pairs. Thus in a 3D reconnection geometry analogous to a 2D $X$-point reconnection geometry, negative (A-type) and positive ( $B$-type) radial nulls are connected by a null-null line intersecting corresponding fan surfaces at the nulls ${ }^{7,8,10}$. This null-null line is called a separator serving as the " $X$-line" on which reconnection takes place, with the fan surfaces serving as the " $X$-arms" (separatrices). This model is called separator reconnection model ${ }^{8,10}$. The existence of such a geometry has been confirmed by in-situ satellite measurements in the magnetosphere ${ }^{5,10,11}$. Similarly, spiral nulls $\left(A_{\mathrm{s}}\right.$ and $B_{\mathrm{s}}$ ) can also be paired by a separator ${ }^{4,6,12}$. Also, it has been shown that multiple null pairs can form clusters. ${ }^{13-16}$ On the other hand, for the $3 \mathrm{D}$ analogy of a 2D $O$-point, as the center of the magnetic island, the null pairs produced by the neutral line breaking are accompanied with the spiral due to the $O$-type geometry and connected by their coincided spine. Torsional spine reconnection then take place at the spiral nulls configuration where "the currents accumulate along the spines and are co-aligned with them" in a recent simulation study ${ }^{16}$. Such numerically predicted spine connected spiral null pair structure is then subject to being tested by observations in space plasmas.

As discussed above, the widely accepted separator model is an analogy of the 2D ' $X$-point' geometry. In literatures, the 'secondary island', corresponding to the ' $O$-point', was 
shown to be important in generating energetic electrons during reconnection ${ }^{17-19}$. Recent 3D simulations show that $3 \mathrm{D}$ flux ropes, rather than $2 \mathrm{D}$ magnetic islands, are expected to be generated during magnetic reconnection ${ }^{14-16,20}$. The flux ropes can interact with each other to lead to complex evolution ${ }^{20}$. Moreover, it is suggested that the secondary reconnection sites may also be present at where the near-null configurations are identified in the flux ropes ${ }^{21}$. In the simulation results in Ref. 14-16, it was illustrated that the flux ropes were related to the spiral nulls, and torsional spine reconnection took place on each spiral null. Similarly, a spiral null point was found to perform as the skeleton of rope structures in the solar active regions ${ }^{12}$. These previous studies imply that the magnetic nulls play an important role in formation of flux ropes. The in-situ observational investigations are necessary to examine previous simulation results and to provide in-depth analysis on the relation between spiral nulls and flux ropes.

In this paper, we show the existence of the spine-paired spiral nulls configuration in space plasmas, observed by Cluster constelation ${ }^{22}$ in the magnetotail. The magnetic configuration is obtained by the fitting-reconstruction method ${ }^{2}$, which reveals that the magnetic structures in the events with 2D secondary island signatures are flux ropes in $3 \mathrm{D}$ geometry, which are formed in close relation with the spine-paired spiral nulls. The kinetic properties and distribution of electrons in the flux ropes are discussed. In Section II, we introduce the instruments and the analysis methods. In Section III, observational and reconstruction results are described. The related kinetic properties and the importance of the spiral null pairs are discussed in Section IV. Section V is the summary.

\section{Data and methods}

In the magnetotail, four Cluster spacecraft are maintained in a shape of approximate regular tetrahedron, giving a chance to investigate the 3D configuration of the reconnection region. The data used in this paper are the magnetic field from $\mathrm{FGM}^{23}$, ion velocity and density form $\mathrm{CIS}^{24}$, electric field and spacecraft potential from $\mathrm{EFW}^{25}$, and electron differential energy flux from PEACE ${ }^{26,27}$. Electron density is derived from the spacecraft potential ${ }^{28}$. The ion initial length $d_{i}$ is calculated according to the local ion density. The 3D electric field is obtained based on the assumption of $E \cdot B=0$, which was applied only 'when 

the magnetic field direction is more than 15 degrees away from the spin plane and $\left|\mathrm{B}_{\mathrm{Z}}\right|$ is larger than $2 \mathrm{nT}$ (otherwise the error in the third electric field component becomes too large)' ${ }^{29}$. The EX $\mathbf{B}$ drift velocities are also calculated under this condition. The electric fields and $\mathbf{E} \times \mathbf{B}$ drift velocities are both smoothed by a smoothing window of $1 \mathrm{~s}$.

The Poincare index ${ }^{30,31}$ is employed to find magnetic null points: $+1(-1)$ means that an A (B)-type or $A_{s}\left(B_{s}\right)$-type null exists in the tetrahedron. In addition, the eigenvalues of the matrix $\delta \mathbf{B}=\partial \mathrm{B}_{\mathrm{i}} / \partial \mathrm{x}_{\mathrm{j}}(\mathrm{i}=\mathrm{x}, \mathrm{y}, \mathrm{z} ; \mathrm{j}=\mathrm{x}, \mathrm{y}, \mathrm{z})$ near the magnetic null are calculated to distinguish the radial and spiral magnetic nulls ${ }^{1,7,8}$. The null is a radial null when all the eigenvalues are real numbers. Otherwise, it is a spiral null. Based on the properties of the eigenvalues, we defined an index called spiral index to identify the spiral nulls. If there is no null or the null is a radial null, the spiral index is zero. Otherwise, the spiral index is set as $+/-1$ to present the $A_{s^{-}} / B_{s^{-}}$ type null (details are given in the supplementary materials ${ }^{32}$ ). In addition, the fitting-reconstruction method ${ }^{2}$ is utilized to obtain the $3 \mathrm{D}$ magnetic field configuration. The details about the method are described in Ref. 2. Benchmark results for the method can be found in Ref. 5. It has been shown that the method has the ability to expose the complex magnetic configuration of separator reconnection. The reconstruction product can capture the topology of the actual structure and gives a creditable result in the regions inside and not too far from $(<3$ times of the size of Cluster tetrahedron) the spacecraft tetrahedron.

In this work, we study a new null point regime in the magnetotail. First, an $A_{\mathrm{s}}$-type null and a $B_{\mathrm{s}}$-type null are connecting by their spine lines. The sketch in Figure 1 illustrates the structure in which two spiral nulls are connected by their common spine. It is modeled as:

$$
\left(B_{x}, B_{y}, B_{z}\right)=\left[x z-\frac{1}{2} j y, y z+\frac{1}{2} j x, 1-z^{2}\right],
$$

where $\mathrm{j}$ is the current density along the spine lines, which leads to the twisted field lines. In this model, two spiral null points locate at $z= \pm 1$, and their common spine lines lay on the $z$ axis, and fan surfaces are perpendicular to the $z$ axis. The field lines around the common spine are twisted exhibiting a flux rope structure. The model with a common spine is thought to be structurally unstable $e^{8,15,16}$. Then by taking a small perturbation of $\delta \mathrm{B}=(\varepsilon z, 0,0)(\varepsilon<<1)$ the model is modified to be:

$$
\left(B_{x}, B_{y}, B_{z}\right)=\left[x z-\frac{1}{2} j y+\varepsilon z, y z+\frac{1}{2} j x, 1-z^{2}\right],
$$


In the modified model shown in Equation (2), the two spiral nulls are no longer connected by their spines. The spine lines of two nulls are separated slightly and helically wrapped together, before exiting near the fan plane of the opposite null from which they were originated. This scenario is the generic situation, with the unperturbed case modeled by Equation (1) where the common spine connects two spiral nulls being a special case. Nevertheless, for weak perturbations $(\varepsilon \ll 1)$, the two helically wrapped spines look nearly straight and are almost overlapped together. Even though the linkages between the two spiral nulls are different in the two models, they can hardly be distinguished in observations. In this paper, we are focusing on how the null points can be paired in the space plasma and its relation to the flux rope formation. In this regards, we call both the unperturbed and perturbed configurations the helically wrapped spine model, with the former and latter being the special and generic case, respectively. In the helically wrapped spine model, the two fan surfaces do not intersect, and the field lines near the spine lines are twisted to form a flux ropes structure, obviously different from the separator model. In the separator model, it is the separator, where the two fans intersect, that connects the two nulls and forms the "X-line" on which reconnection occurs. To ensure that the fitting-reconstruction method is applicable to the configuration studied in this paper based on data from Cluster measurements, additional benchmark is done. The benchmark results are presented in the supplemental materials ${ }^{32}$ of this paper. It shows that the reconstruction results are able to capture the essential characteristics of the helically wrapped spine model. In this paper, we only qualitatively analyze the reconstruction results, rather than to quantitatively study the details of the reconstructions.

\section{Observational and reconstruction Results}

On September 15 2001 around 05:03 UT, Cluster travelled into the magnetic reconnection region in the magnetotail ${ }^{1,2}$. The main measurements are shown in Figure 2 in the GSM coordinate, in which the $X$-axis is in the direction pointing from Earth to the Sun, and $Z$-axis parallel to the magnetic dipole axis, i.e., the magnetic north. In the magnetotail, the $X$-axis is mainly pointing to the Earth. The $X$-component of ion velocity $V_{X}$ observed by $\mathrm{C} 1$ changed its sign from positive to negative (Figure 2a) around 05:03:35 UT, while the sign of magnetic component $B_{Z}$ (Figure $2 \mathrm{~b}$ ) altered from positive to negative as well (the slight 
difference between the $V_{X}$ and $B_{Z}$ reversal periods could be due to the rotation of the current sheet). It implies that the spacecraft encountered a reconnection region and moved from the earthward outflow region to the tailward outflow region. In the earthward outflow region, $\mathrm{C} 1$ detected a bipolar signal of $B_{Z}$ (illustrated by the brown shadow), indicating that a structure with secondary island signature had been formed there. In literatures similar structures were called "secondary islands" 18,19. For clarification, it should be pointed out that the secondary island is a two-dimensional (2D) concept, while the structures observed are three-dimensional (3D) ones. Therefore, while using the term of the "secondary island", it only means that the structure has secondary-island-like observable features. Distinct from $\mathrm{C} 1$, the other three satellites did not observe the $B_{Z}$ bipolar signal. $\mathrm{C} 4$ was in the north of $\mathrm{C} 1$, while $\mathrm{C} 3$ was in the south of them. This suggests that the secondary island had a size smaller than the spacecraft separation $\left(\sim 2000 \mathrm{~km}\right.$, while $d_{i} \sim 1020 \mathrm{~km}$ for $n \sim 0.05 \mathrm{~cm}^{-3}$ obtained from CIS on board C1). An electron density minimum was measured by $\mathrm{C} 1$ (Figure 2c) when the spacecraft was passing across the trailing edge of the structure with secondary island signature where the flux of electrons with energies larger than $1 \mathrm{keV}$ (hot electrons) was decreased as well (Figure 2d).

The Poincaré index in Figure 2e shows that magnetic nulls existed inside the structure with secondary island features; and the spiral index implies that the magnetic nulls were spiral types. Figure 3 displays two reconstruction results during $\mathrm{C} 1$ was passing through the structure. The two moments of the reconstructions are marked by the black dashed lines in Figure 2. The skeleton of the magnetic structure shown in Figure $3 \mathrm{a}$ is reconstructed at 05:03:24.296 UT. It reveals that the secondary-island-like structure is consisted of two spiral nulls. The $A_{s}$-type null locates at $\sim[50,-210,0] \mathrm{km}$, and the $B_{s}$-type null is at $\sim[320,1170$, -210] $\mathrm{km}$ (where the original point is at the center of the tetrahedron). The field lines adjacent to the two spiral nulls are plotted as the colored curves with arrows. The colors represent the magnitude, and the arrows denote the field line orientation. Spiral field lines manifest the fan surfaces. The field line bundles are roughly perpendicular to the fan surfaces, unveiling the spine lines. C1 and C2 (black and red small spheres) are on the fan surfaces of the $A_{s}$-null and $B_{s}$-null respectively. The interesting feature is that a spine line exists in between the two spiral nulls with a length of $\sim 1420 \mathrm{~km}$. The magnetic configuration is consistent with the 
helically wrapped spine model discussed in Section II. In the reconstruction result, it is very hard to tell whether the $A_{s}$ and $B_{s}$ nulls are connected by a common spine, or the two nulls connect with different spines that wrap each other closely. It is needed to develop a more powerful method to distinguish these two configurations. The two fan surfaces in Figure 3a are roughly in the $x-z$ plane, and the spine lines are mainly in the $Y$-direction, i.e., almost the out-of-plane direction. Figure $3 \mathrm{~b}$ presents the reconstruction result at 05:03:24.965 UT. It exhibits similar features as in Figure 3a. A pair of spiral nulls look like interlinked by their spine lines, too. The magnetic field lines in the vicinity of the spine lines are plotted as thick purple curves. This shows that these field lines rotate around the spine lines to form the flux ropes, presenting the "secondary island" feature in the 2D view. The two fan surfaces separate the flux ropes in three regions. The thick purple twisted curves illustrate the flux rope with the spines being embedded inside ${ }^{16}$. On the other sides of both fan planes, there are two more flux ropes extending outside of the reconstruction region. We cannot get the total length of the three flux ropes for the limited ability of the fitting-reconstruction method to reconstruct field too far from the tetrahedron. What we can obtain is the width of the flux rope in the tetrahedron, which is estimated to be $\sim 1200 \mathrm{~km}$ in the $X$-direction (which is roughly the outflow direction). $\mathrm{C} 3$ and $\mathrm{C} 4$ are outside of the edge of the flux ropes. As a consequence, the two satellites did not record the $B_{Z}$ reversal signal. C2 did not obtain this signal either, which will be discussed in the next section.

To investigate the kinetic properties near the two nulls, EXB drift velocities at $\mathrm{C} 1$ and $\mathrm{C} 2$ are presented in Figures $2 \mathrm{f}$ and $2 \mathrm{~g}$. The corresponding electric field measurements demonstrate that large $E_{\mathrm{Z}}$ is detected by $\mathrm{C} 1$ and $\mathrm{C} 2$ (shown in Figures $2 \mathrm{~h}$ and $2 \mathrm{i}$ respectively), which should be the Hall electric field pointing toward to the current sheet center ${ }^{33,34}$. Before the Poincaré index changes to +1 , the $\mathbf{E} \times \mathbf{B}$ drifts at $\mathrm{C} 1$ and $\mathrm{C} 2$ hold an obvious $Y$-component with a magnitude of $-2500 \mathrm{~km} / \mathrm{s}$, implying that a large flow exists in the out-of-plane direction. Around the time when the Poincaré index starts to be nonzero, the spiral nulls begin to appear in the reconstruction results. During the time when the spiral null pair is reconstructed, the $\mathbf{E} \times \mathbf{B}$ drifts at $\mathrm{C} 2$ is decreased to $\sim 500 \mathrm{~km} / \mathrm{s}$. Unfortunately the data from $\mathrm{C} 1$ are not good enough to calculate the 3D electric field and obtain the EXB drifts. The reconstruction results in Figure 3 show that $\mathrm{C} 2$ is on the fan surface of the $B$-type null, while 
the normal of this fan surface is $[0.35,-0.94,-0.04], \sim 20^{\circ}$ deviating from the $Y$-direction, and intersecting the spine line with an angle of $\sim 30^{\circ}$. Thus, the $\mathbf{E} \times \mathbf{B}$ drifts at $\mathrm{C} 2$ suggest that magnetized electrons flow across the fan surface of the $B_{s}$-null and may have a significant component co-aligned with the spine lines, as suggested by the simulation results in Ref. 16 .

The helically wrapped spine model configuration is also found in the event at $~ 05: 01: 25$ UT on September $15^{\text {th }} 2001$, as presented in Figure 4. Similar to the first event, a structure with secondary island signatures is illustrated by Bz bipolar (Figure 4a), and the spiral index (Figure 4b) implies that Cluster encountered spiral nulls. The $A_{\mathrm{s}}$-spine- $B_{\mathrm{s}}$-like configuration shown in Figure $4 \mathrm{e}$ is obtained by fitting-reconstruction at 05:01:21.781 UT (marked by black dashed line in Figures $4 \mathrm{a}-4 \mathrm{~d})$. The $A_{s}$-null is at $\sim[160,-920,-740] \mathrm{km}$, and the $B_{s}$-null is at $\sim[-690,690,-850] \mathrm{km}$. The length of the spine in between the two spiral nulls is $\sim 1820$ $\mathrm{km}$. The field line near the spine line is wrapped to form the flux rope (see the thick purple line in Figure 4e), which has a secondary island geometry in 2D view. The fan surface of $A_{s}$-null tilts from the $\mathrm{x}-\mathrm{z}$ plane, leading to the bipolar signal of $\mathrm{B}_{\mathrm{y}}$ as well. During this interval, the electron density was not obtained from the spacecraft potential because that the ASPOC instrument was operating. Instead, the proton density detected by CIS-CODIF is plotted in Figure 3c. It shows that plasma density decreases in the flux rope, similar to the first event. Contrary to the first event, the flux of hot electrons is enhanced in the flux rope (Figure 4d), which will be discussed in the next section.

\section{Discussion}

In the last Section, we presented two secondary island events observed by Cluster in the magnetotail. The secondary island signatures shown in the events were both measured in the outflow region. The reconstruction results reveal that in 3D the "secondary islands" observed are flux ropes related to two spiral nulls paired via their spine lines. To distinguish it from the separator model, we call the null pair model analyzed in this paper as the helically wrapped spine model. We have reconstructed similar structures in another two magnetotail reconnection events in relation with observation of secondary island signatures (at 05:05:26 UT on September 15, 2001, and at 09:48:42 UT on October 01, 2001), which are not illustrated in this paper to avoid redundancy. We note that particle-in-cell simulation of 
reconnection in a cluster of null points showed alike structures ${ }^{16}$. Therefore, the helically wrapped spine model appears to be a potentially important model to form flux ropes. One then needs to pay more attentions to the $A_{\mathrm{s}}$-spine- $B_{\mathrm{s}}$-like configuration, which has barely been discussed before. In this section, we will compare our observations with previous studies and models to illuminate the role of the helically wrapped spine model in formation of flux ropes.

\section{A. Linkage between magnetic nulls}

In general, two nulls with different polarities can be connected by a null-null line as (i) the intersection of two fan surfaces, (ii) the spine of one null on the fan of the other null, and (iii) the spine lines of both nulls ${ }^{8}$. Case (i) is seen in the well-known separator model. Only the separator model can replicate the 2D $X$-type topology on every plane intersecting and perpendicular to the null-null line. In general, structures in both Case (ii) and Case (iii) are geometrically unstable ${ }^{8}$. Both simulation studies in Ref 16 and our observation/reconstruction show structures similar to Case (iii) that two spiral nulls may be interlinked by their spine lines. The major difference between the spiral and radial nulls is whether there is noticeable current $\left(j_{\|}\right)$along the spine line. When $j_{\|}$exceeds a critical value, the radial magnetic null will change to a spiral null, with twisting field lines around the null ${ }^{7-9}$.

More than two nulls can be assembled together to form null clusters ${ }^{13-16}$. The specific way to connect nulls can largely control the topology of the null cluster. The simulation results in Ref. 16 show that torsional spine reconnection takes place on each spiral null of a cluster of spiral nulls chained by spine lines. Between some null point pairs, their fan surfaces diverge away from others. Meanwhile, in some other places, the fan surfaces of two null points are intersected to form separators, when the secondary bifurcation takes place ${ }^{15}$. In all cases, near the spine lines, each pair of nulls in the spiral null chain has the configuration similar to the helically wrapped spine model shown in our paper. Even though the $A_{s}$-spine- $B_{s}$ geometry is structurally unstable ${ }^{8}$, as we mentioned in Section II and the simulation ${ }^{16}$ showed, at least when perturbations are weak, the perturbed configurations remain similar to the unperturbed one. The magnetic field lines wrap around the spine lines to form flux ropes. The 2D $O$-type topology of magnetic islands is seen on the plane perpendicular to the spine lines. 
Different connection type of two null points leads to entirely different magnetic topology. The separator model is corresponding to the $X$-line where reconnection primarily occurs ${ }^{35,5}$. The helically wrapped spine model is matched to the $O$-line which is referred to as the secondary island in the 2D approximation. Multiple magnetic nulls can be detected in the magnetosphere ${ }^{1,4}$, magnetosheath ${ }^{6}$, and solar atmosphere ${ }^{12}$. Our results suggest that the null-null line interlinking a pair of nulls among the null clusters is not only the intersecting field line of two fan surfaces predicted by the separator model, but might also be the spine lines of two spiral nulls, as shown in Figure 1.

\section{B. spiral nulls related flux ropes}

The observed structures with secondary island signatures in this paper are flux ropes. The reconstruction results in Figures 3 and $4 \mathrm{e}$ indicate that the flux rope is formed in close association with a pair of $A_{s}$-type and $B_{s}$-type nulls. The two spiral nulls are paired via their spine lines. The flux rope can also be generated through other configurations with/without spiral null points as well, which will be discussed in our subsequent work. The simulation results in Ref. 16 also show that multiple spiral null points interconnected via spine lines embed in flux ropes and form a null chain. Our reconstruction results may just be a part of the spiral null chain. Unfortunately, our benchmark results ${ }^{5}$ showed that the reconstruction results are reliable only inside and not far away from the spacecraft tetrahedron. Therefore, it is unfeasible to verify whether the flux ropes were composed of only a spiral null pair, or a chain of spiral nulls.

Various electron characteristics have been observed inside the flux rope in this work. The electron fluxes in flux rope are low in the first event (Figure 2), while hot electron flux is enhanced in the flux rope in the second event (Figure 4). Additionally, the electron density does not enhance in the flux rope in both cases, different from previous study ${ }^{18}$. The differences in different events could be related to the formation of flux rope. If the flux rope is produced in the outflow region locally (detailed in subsection C.), the plasma characteristics can show different features of different generation regions with diverse properties. Furthermore, as shown in Ref.16, more than two spiral nulls could be linked together to form a chain. The spiral null chain would connect two regions with various 
characteristics and make the flux rope much complicated.

\section{Magnetic reconnection in the flux ropes}

The helical field lines surrounding the spiral nulls are principally caused by the intense current tangential to the spine lines, as suggested by our model given in Figure 1 and seen in the simulation results ${ }^{16}$. In such configurations, reconnection may be classified as torsional spine reconnection based on previous MHD theory ${ }^{8,9}$. Recently, 3D simulation results show that the flux ropes in reconnection exhaust far from the primary reconnection site can host secondary reconnection sites, suggesting that 'secondary reconnection occurs in a large part of the exhaust' ${ }^{21}$. The flux ropes in that simulations are similar to the spiral null pair structures shown in this paper.

For the first event, the pair of the spiral nulls in our reconstruction was created in the reconnection outflow region locally. It may be formed just a few seconds before the reconstruction period shown in Figure 3. The reason is that the decrease of ion velocity (shown in Figure 2a) implies that the spacecraft were traveling from the earthward side of reconnection region to the tailward outflow region. $\mathrm{C} 1$ was at the earthward of all other three satellites, as can be found in Figure 3 (the spacecraft are marked as small colored spheres). If the flux rope was formed before Cluster passed over it, other spacecraft might all detect the bipolar $B_{Z}$ variations prior to $\mathrm{C} 1$ when traversing through the flux rope. The fact that only $\mathrm{C} 1$ encountered the bipolar $\mathrm{Bz}$ indicates that the most possible scenario is that the spiral null pair and the flux rope were newly created between $\mathrm{C} 1$ and $\mathrm{C} 2$ (C2 was earthward of $\mathrm{C} 3$ and $\mathrm{C} 4$, and tailward of C1), locally in the spacecraft tetrahedron region. This scenario is consistent with the fact that spiral nulls are start to be uncovered by reconstruction around the time when Poincaré index became nonzero and $\mathrm{Bz}$ became negative. In conclusion, we observed a locally generated flux rope and the associated a pair of spiral nulls in the magnetotail reconnection outflow region, which is consistent with the $3 \mathrm{D}$ simulation ${ }^{21}$ that the reconnection exhaust away from the primary reconnection site may become host to secondary reconnection sites.

The $\mathbf{E} \times \mathbf{B}$ drifts detected by $\mathrm{C} 1$ and $\mathrm{C} 2$ near the fan surfaces in the first event indicate 
that the magnetized electrons streamed in the out-of-plane direction, i.e., mainly in the $Y$-direction. Besides the $Y$-component of the drift velocity, the $X$ - and $\mathrm{Z}$-components were noticeable as well. In torsional spine reconnection, the magnetized electrons drift on the fan surfaces at the $\mathbf{E} \times \mathbf{B}$ drift velocity ${ }^{9}$. Such drifts around the spines of spiral nulls have been found in 3D reconnection in the turbulent magnetosheath ${ }^{6}$. Besides, in spine-fan reconnection, there is drift across the fan surface ${ }^{9}$. Indicating by the wrapped field lines and non-perpendicularity between spine and fan surface, both the torsional-spine and spine-fan reconnection take place in this event. Field lines and magnetized electrons rotate about the spine, and meanwhile traverse the fan surfaces in the out-of-plane direction, implied by the component of $\mathbf{E} \times \mathbf{B}$ drift perpendicular to the fan surfaces.

\section{Summary}

The helically wrapped spine model with an $A_{s}$ and $B_{s}$ null pair configuration in 3D reconnection is observationally studied based on Cluster multiple-spacecraft measurements. Different from the separator model, this null pair structure provides an additional way to pair two spiral null points via their spine lines. In the separator model, the $X$-type topology can be seen on the plane perpendicular to the separator. Distinctly, in the helically wrapped spine model presented in our study, the $O$-type topology is seen on the plane perpendicular to the spine. Similar to previous studies ${ }^{12,14-16}$, the reconstruction results show that in $3 \mathrm{D}$ the $O$-point configurations manifest flux ropes rather than closed field line islands in nature. The field lines are twisted around the spine lines to form flux ropes. Furthermore, implying by the ExB drift, magnetized electrons rotate on and cross the fan surface, suggesting that torsional-spine and spine-fan reconnection both take place in the configuration ${ }^{8,9,16}$, and in agreement with the 3D PIC simulations ${ }^{16}$. In addition, for the first event, detailed analysis shows that the spiral null pair and flux rope are newly formed in a local outflow region. This indicates that reconnection exhaust away from the primary reconnection site may become host to secondary reconnection sites, delivering the accordant statement with the simulation results ${ }^{21}$.

One of the poorly understood issues of 3D reconnection is where reconnection takes 
place and how it involves. Magnetic nulls (both radial and spiral) are frequently observed in the reconnection region ${ }^{1-6,10}$. Previous works have shown that null points and flux ropes are essential factors to participate in reconnection ${ }^{5,10,12,14,15,20}$. It was shown in Ref. 20 that 'the three-dimensional evolution is dominated by the formation and interaction of helical magnetic structures known as flux ropes'. Cluster observations of $A_{s}$-spine- $B_{s}$-like configuration and helically wrapped spine model investigated in our work further illustrate that flux ropes can be involved in torsional spine reconnection in the magnetospheric environment, such as the magnetotail exhaust, which can be identified by newly operational MMS mission.

\section{Acknowledgements}

This work is supported by the Chinese NSFC programs 41274167, 41374166, 41474139, 41404117 and PKU/UCLA JRI, and also partly by the ESA 2013-2014 Guest Investigator Program and a working group sponsored by ISSI, Berne.. We acknowledge the Cluster PEACE, CIS, FGM and EFW instrument teams for the provision of the data and the Cluster CSA for providing access to the data. M Dunlop and Y V Bogdanova are supported by STFC in-house research grant. We thank the International Space Science Institute in Bern, Switzerland, its staff and directors for partial support through the ISSI Team (from Cluster to MMS).

\section{References}

${ }^{1}$ C. J. Xiao, X. G. Wang, Z. Y. Pu, H. Zhao, J. X. Wang, Z. W. Ma, S. Y. Fu, M. G. Kivelson, Z. X. Liu, Q. G. Zong, K. H. Glassmeier, A. Balogh, A. Korth, H. Reme And C. P. Escoubet, Nat. Phys., 2, 478, (2006).

${ }^{2}$ J.-S. He, C.-Y. Tu, H. Tian, C.-J. Xiao, X.-G. Wang, Z.-Y. Pu, Z.-W. Ma, M. W. Dunlop, H. Zhao, G.-P. Zhou, J.-X. Wang, S.-Y. Fu, Z.-X. Liu, Q.-G. Zong, K.-H. Glassmeier, H. Reme, I. Dandouras, and C. P. Escoubet, J. Geophys. Res., 113, A05205 (2008a).

${ }^{3}$ J.-S. He, Q.-G. Zong, X.-H. Deng, C.-Y. Tu, C.-J. Xiao, X.-G. Wang, Z.-W. Ma, Z.-Y. Pu, E. Lucek, A. Pedersen, A. Fazakerley, N. Cornilleau-Wehrlin, M. W. Dunlop, H. Tian, S. Yao, B. Tan, S.-Y. Fu, K.-H. Glassmeier, H. Reme, I. Dandouras, and C. P. Escoubet, Geophys. Res. Lett., 35, L14104 (2008b). 
${ }^{4}$ X. H. Deng, M. Zhou, S. Y. Li, W. Baumjohann, M. Andre, N. Cornilleau, O. Santolı' k, D. I. Pontin, H. Reme, E. Lucek, A. N. Fazakerley, P. Decreau, P. Daly, R. Nakamura, R. X. Tang, Y. H. Hu, Y. Pang, J. Bu“chner, H. Zhao, A. Vaivads, J. S. Pickett, C. S. Ng, X. Lin, S. Fu, Z. G. Yuan, Z. W. Su, and J. F. Wang, J. Geophys. Res., 114, A07216 (2009).

${ }^{5}$ R. L. Guo, Z. Y. Pu, C. J. Xiao, X. G. Wang, S. Y. Fu, L. Xie, Q. Z. Zong, J. S. He, Z. H. Yao, J. Zhong, and J.X. Li, J. Geophys. Res., 118, 6116 (2013).

${ }^{6}$ D. E. Wendel, and M. L. Adrian, J. Geophys. Res., 118, 1571 (2013).

${ }^{7}$ Y.-T. Lau , and J. Finn, Astrophys. J., 350, 672 (1990).

${ }^{8}$ E. R. Priest, and V. S. Titov, Phil. Trans. R. Soc. London. A, 354, 2951 (1996).

${ }^{9}$ E. R. Priest, and D. I. Pontin, Phys. Plasmas, 16, 122101 (2009).

${ }^{10}$ C. J. Xiao, X. G. Wang, Z. Y. Pu, Z. W. Ma, H. Zhao, G. P. Zhou, J. X. Wang, M. G. Kivelson, S. Y. Fu, Z. X. Liu, Q. G. Zong, M. W. Dunlop, K.-H. Glassmeier, E. Lucek, H. Reme, I. Dandouras And C. P. Escoubet, Nat. Phys., 3, 609 (2007).

${ }^{11}$ M. W. Dunlop, Q. H. Zhang, C. J. Xiao, J. S. He, Z. Y. Pu, R. C. Fear, C. Shen, and C. P. Escoubet, Phys. Rev. Lett., 102(7), 075005 (2009).

${ }^{12}$ H. Zhao, J.-X. Wang, J. Zhang, C.-J. Xiao and H.-M. Wang, Chin. J. Astron. Astrophys. 8, No. 2, 133 (2008).

${ }^{13}$ J. C. Dorelli, A. Bhattacharjee, and J. Raeder, J. Geophys. Res., 112, A02202 (2007).

${ }^{14}$ P. F. Wyper and D. I. Pontin, Physics of Plasmas, 21, 082114 (2014a).

${ }^{15}$ P. F. Wyper and D. I. Pontin, Physics of Plasmas, 21, 102102 (2014b).

${ }^{16}$ V. Olshevsky, A. Divin, E. Eriksson, S. Markidis, and G. Lapenta, The Astrophysical Journal, 807:155 (2015).

${ }^{17}$ J. F. Drake, M. Swisdak, H. Che, and M. A. Shay, Nat. Phys., 443, 553 (2006).

${ }^{18}$ L.-J. Chen, A. Bhattacharjee, P. A. Puhl-Quinn, H. Yang, N. Bessho, S. Imada, S. Mu“ Hlbachler, P. W. Daly, B. Lefebvre, Y. Khotyaintsev, A. Vaivads, A. Fazakerley And E. Georgescu, Nat. Phys., 4, 19 (2008).

${ }^{19}$ R. S. Wang, Q. M. Lu, A. M, Du, and S. Wan, Phys. Rev. Lett., 104, 175003 (2010).

${ }^{20}$ W. Daughton, V. Roytershteyn, H. Karimabadi, L. Yin, B. J. Albright, B. Bergen and K. J. Bowers, Nature Physics, 7, 539(2011)

${ }^{21}$ G. Lapenta, S. Markidis, M. V. Goldman and D. L. Newman, Nature Physics, 11, 690 (2015).

${ }^{22}$ C. P. Escoubet, M. Fehringer and M. Goldstein, Ann. Geophys., 19, 1197 (2001).

${ }^{23}$ A. Balogh, C. M. Carr, M. H. Acũna, M. W. Dunlop, T. J. Beek, P. Brown, K.-H. Fornacon, E. Georgescu, K.-H. Glassmeier, J. Harris, G. Musmann, T. Oddy, and K. Schwingenschuh, Ann. Geophys., 19, 1207 (2001).

${ }^{24}$ H. R`eme et al., Ann. Geophys., 19, 1303 (2001).

${ }^{25}$ G. Gustafsson, R. Bergstr"om, B. Holback et al., Space Sci. Rev., 79, 137 (1997).

${ }^{26}$ A. D. Johnstone And C. Alsop, S. Burge , P. J. Carter, A. J. Coates, A. J. Coker And A. N. Fazakerley , M. Grande , R. A. Gowen , C. Gurgiolo , B. K. Hancock , B. Narheim , Space Sci. Rev., 79, 351 (1997).

${ }^{27}$ A. Fazakerley, A. Lahiff, R. Wilson, I. Rozum, C. Anekallu, M. West, and H. Bacai, PEACE data in the Cluster active archive, in The Cluster Active Archive, pp. 129-144, Springer (2010).

${ }^{28}$ A. Pedersen, B. Lybekk, M. Andre', A. Eriksson, A. Masson, F. S. Mozer, P.-A. Lindqvist, P. 

M. E. De'cre'au, I. Dandouras, J.-A. Sauvaud, A. Fazakerley, M. Taylor, G. Paschmann, K. R. Svenes, K. Torkar, and E. Whipple, J. Geophys. Res., 113, A07S33 (2008). ${ }^{29}$ P. A. Lindqvist, C. Cully, Y. Khotyaintsev, and the EFW team, in User Guide to the EFW Measurements in the Cluster Active Archive (CAA), Cluster Active Archive. (2013)

J. M. Greene, J. Comput. Phys., 98, 194 (1992).

${ }^{32}$ See supplemental material at [URL will be inserted by AIP] for Spiral Index and benchmark of the fitting-reconstruction method

${ }^{33}$ J. P. Eastwood, T.-D. Phan, F. S. Mozer, M. A. Shay, M. Fujimoto, A. Retino`, M. Hesse, A. Balogh, E. A. Lucek, and I. Dandouras, J. Geophys. Res., 112, A06235 (2007).

${ }^{34}$ L.-J. Chen, W. Daughton, A. Bhattacharjee, R. B. Torbert, V. Roytershteyn, and N. Bessho, Physics of Plasmas, 19, 112902 (2012). 


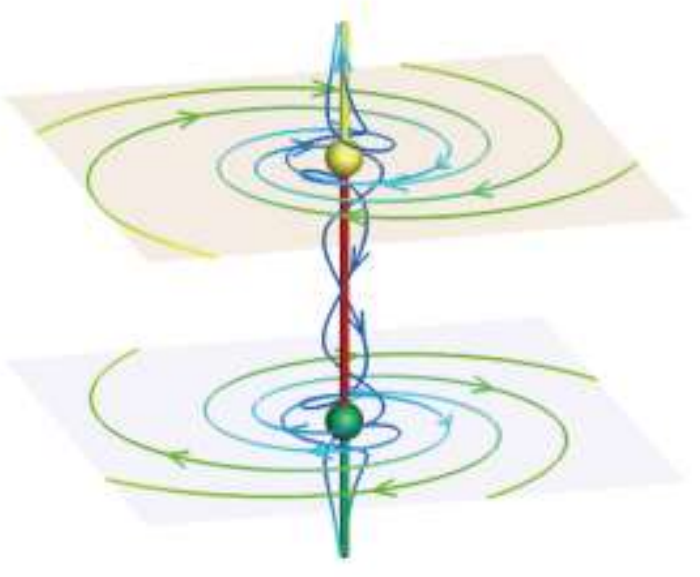

455 FIG. 1. Illustration for the helically wrapped spine model $\left(\left(B_{x}, B_{y}, B_{z}\right)=\left[x z-\frac{1}{2} j y, y z+\frac{1}{2} j x, 1-z^{2}\right]\right)$.

456 The yellow sphere is As-type null and the green sphere is Bs-type null. Red line is the 457 common spine linked two nulls. Yellow and green lines are spines of As- and Bs- nulls

458 respectively. Color curves with arrows are field lines. Light brown plane is the fan surface of 459 As-type null, and light blue plane is the fan surface of Bs-type null. The field lines around the 460 common spine are twisted to form flux ropes. 


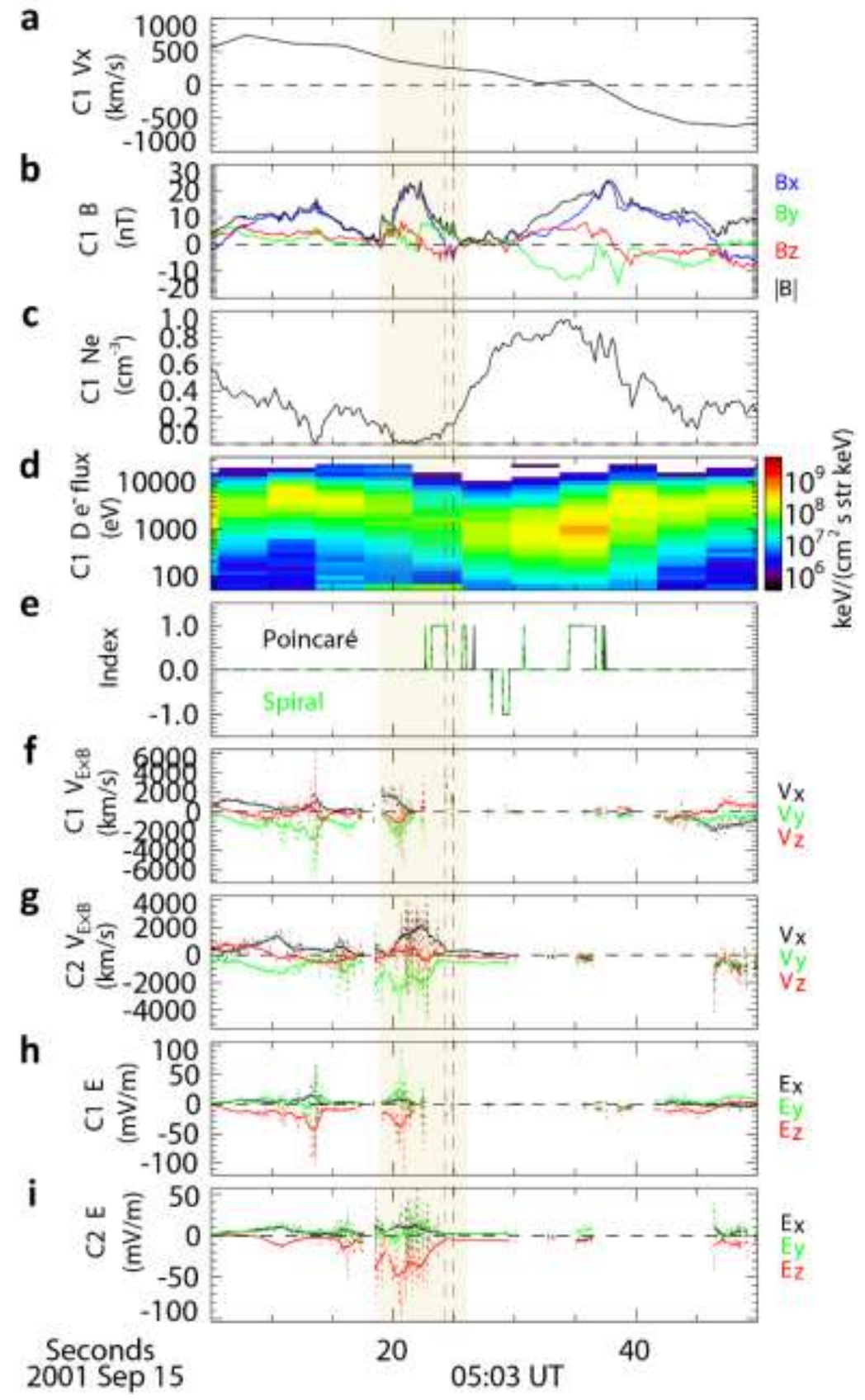

FIG. 2. The first secondary island event observed by Cluster on September 15, 2001. (a) Ion velocity from CIS-HIA for C1. (b) Magnetic field vector and strength for C1. (c) Electron density derived from spacecraft potential for C1. (d) Differential energy flux of electrons accumulated from all pitch-angles for C1. (e) Black line is Poincaré index, and dashed green line is spiral index. If both two indexs are +/- 1 , As-/Bs- type null exists in Cluster tetrahedron. If only Poincaré index is +/- 1 while spiral index is 0 , the null is $A-/ B-$ type null. (f) ExB drift velocity for C1 and (g) for C2. (h) Electric field for C1 and (i) for C2. The dotted lines in ( $f-i)$ are the original data provided by CSA, and the solid lines are the smoothed results of the original data. The smooth window is one second. Brown mask marks out the interval when Bz showed bipolar signal. Two dashed vertical lines mark the times to do reconstructions. The coordinate for all vectors is the GSM coordinate. 


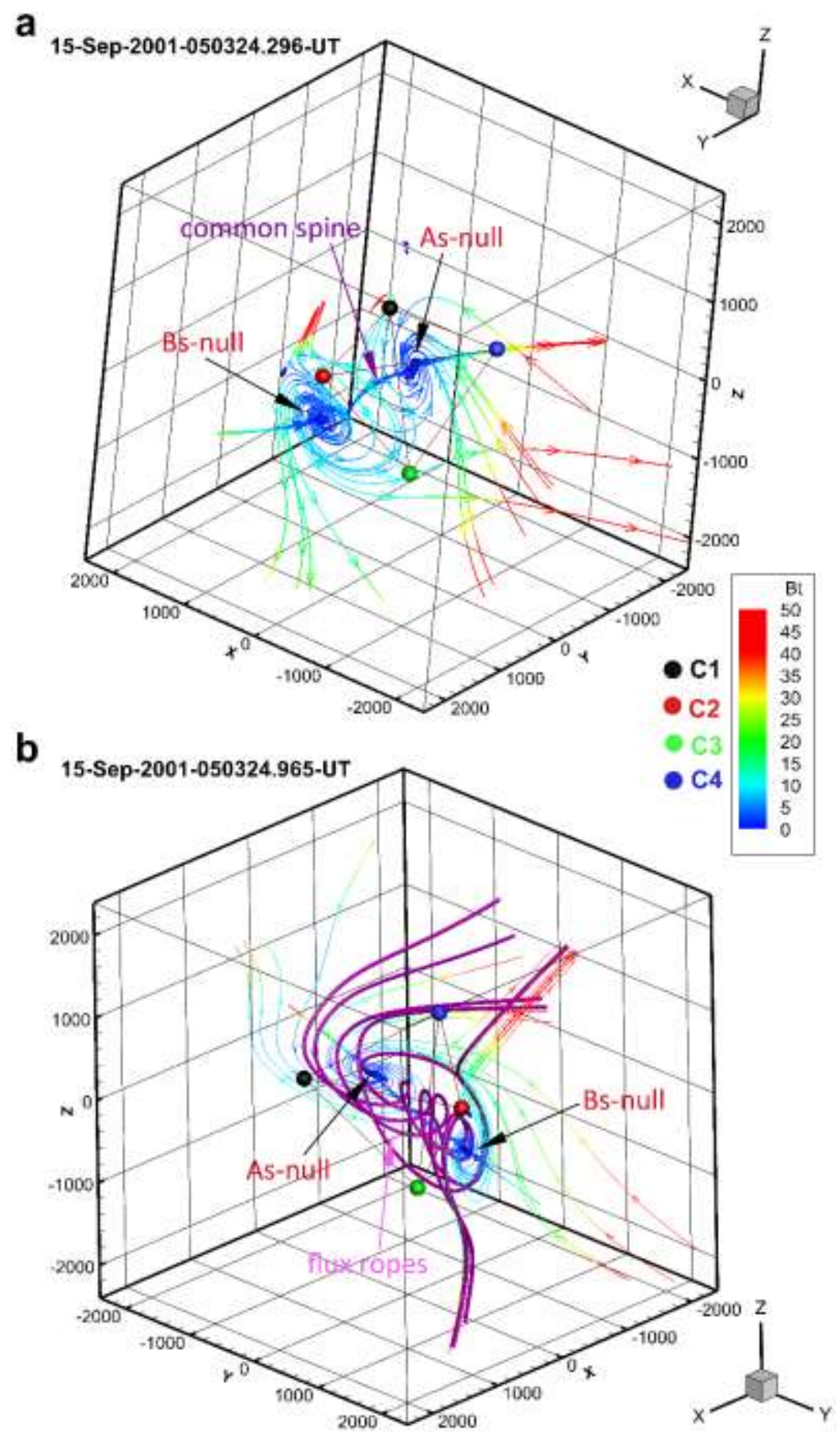

FIG. 3. Reconstruction results for the two times marked by the dashed lines in Figure 2. (a) Magnetic configuration reconstructed at 05:03:24.296 UT. Colored spheres present the location of four Cluster satellites (Black, red, green and blue represent $\mathrm{C} 1-\mathrm{C} 4$ respectively). Colored curves are constructed magnetic field lines. The arrows on the curves show the direction of the field lines. The configuration consists of a Bs-null and an As-null. The two spiral nulls are interlinked by their spine which directs approximately to the Y-direction. (b) Magnetic configuration reconstructed at 05:03:24.965 UT. The configuration gives the similar structure as in (a). The thick purple curves are also field lines, which are plotted to show the flux ropes. 

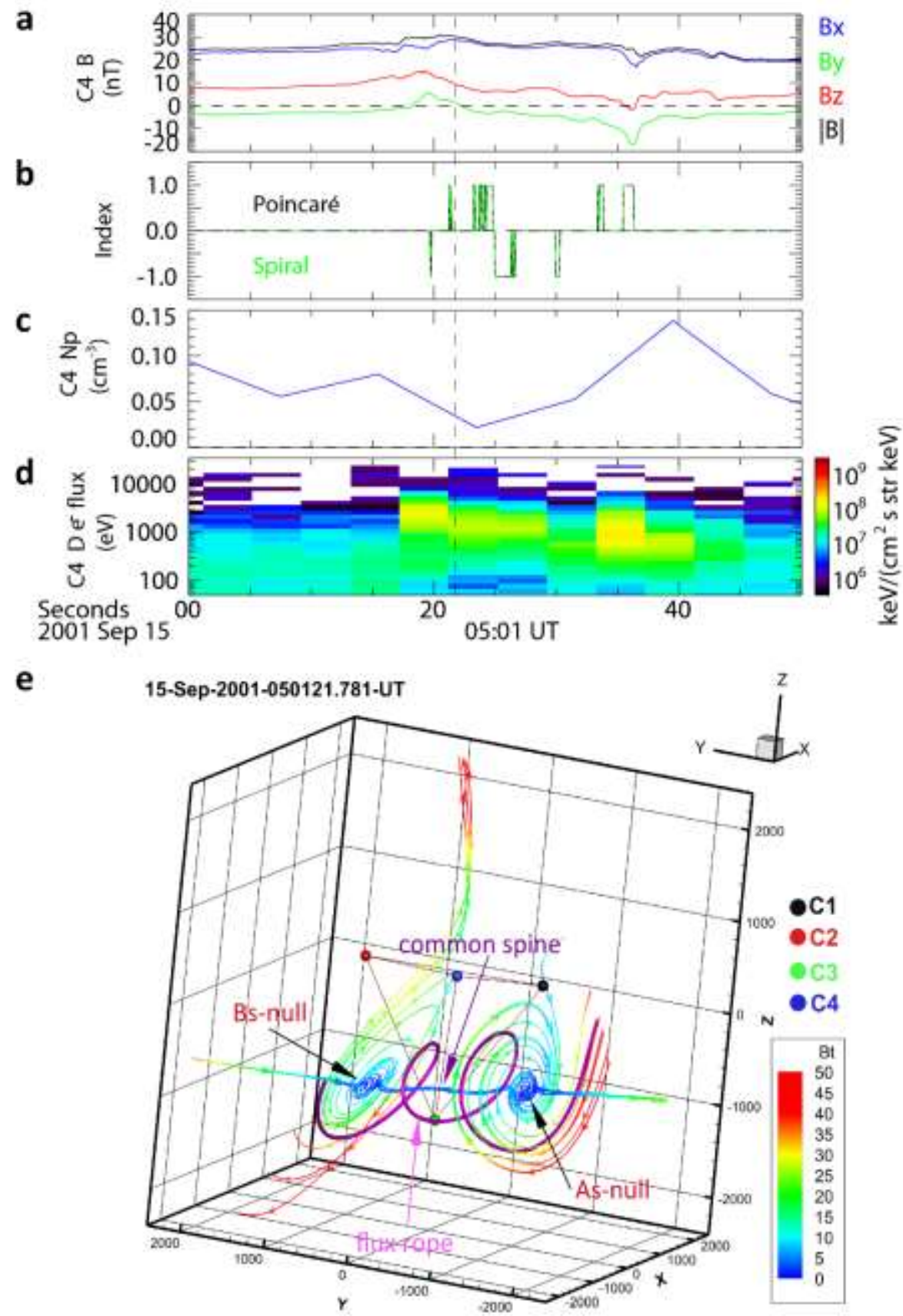

FIG. 4. The second secondary island event observed by Cluster on September 15, 2001. (a) Magnetic field vector and strength for C4. (b) Black line is Poincaré index, and dashed green line is spiral index. If both two indexs are +/- 1, As-/Bs- type null exists in Cluster tetrahedron. If only Poincaré index is +/- 1 while spiral index is 0 , the null is A-/B- type null. (c) Proton density observed by CIS-CODIF on board C4. (d) Differential energy flux of electrons accumulated from all pitch-angles for $\mathrm{C} 4$. (e) Reconstruction results for the time at 05:01:21.781 UT. Colored spheres present the location of four Cluster satellites (Black, red, green and blue represent $\mathrm{C} 1-\mathrm{C} 4$ respectively). Colored curves are constructed magnetic field lines. The arrows on the curves show the direction of the field lines. The configuration consists of a Bs-null and an As-null. The two spiral nulls are interlinked by their spine which directs approximately to the Y-direction. The thick purple curve is field line to show the flux rope. 


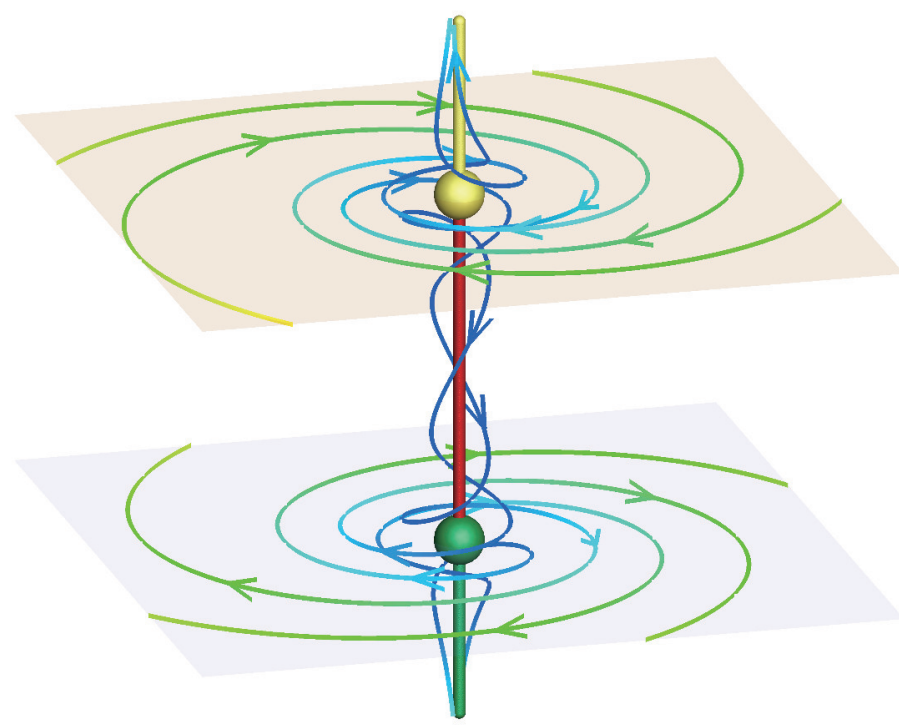





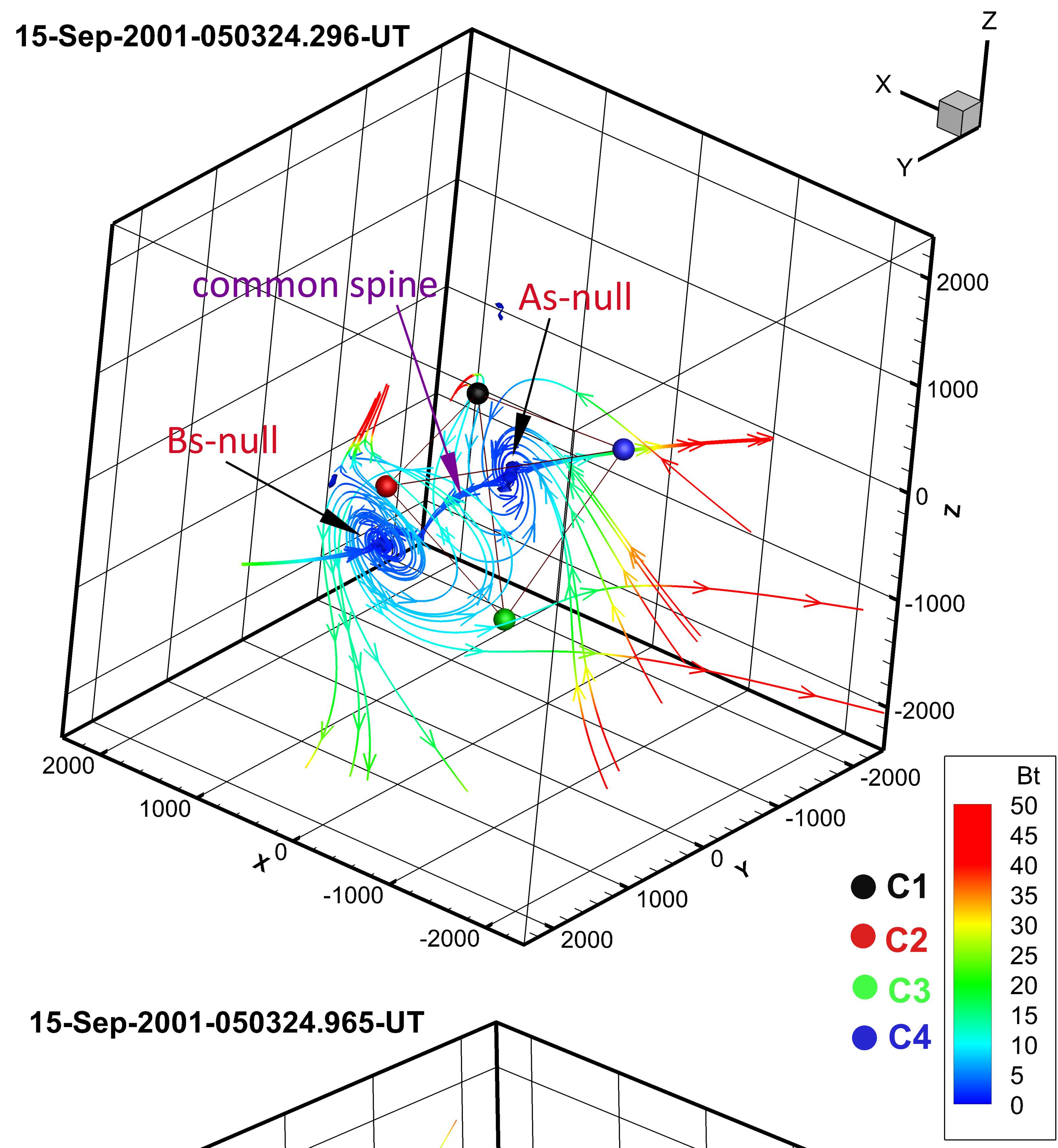

b

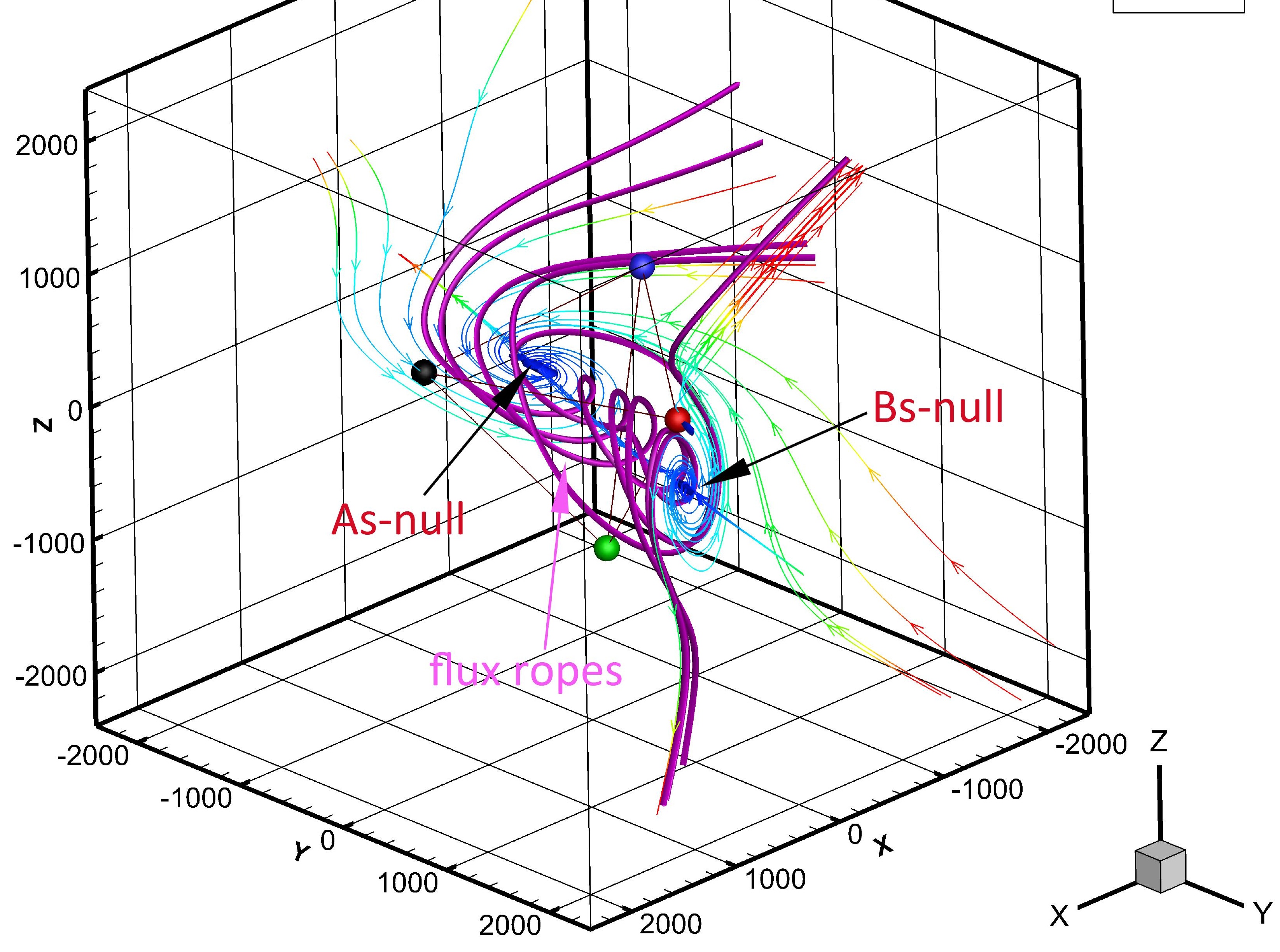


$\mathbf{a}$

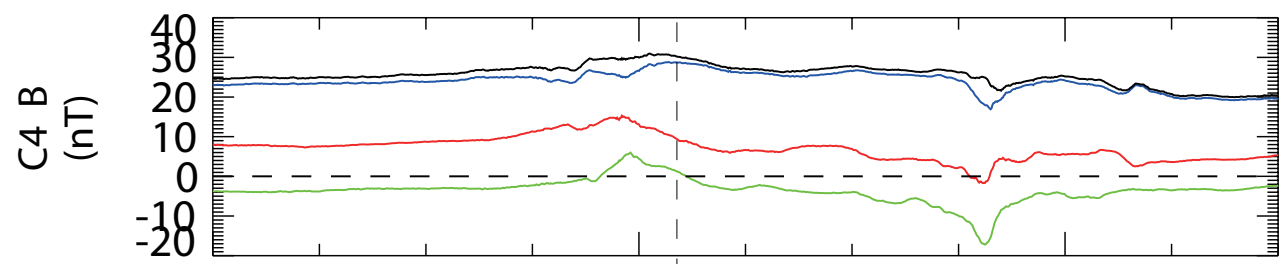

Bx

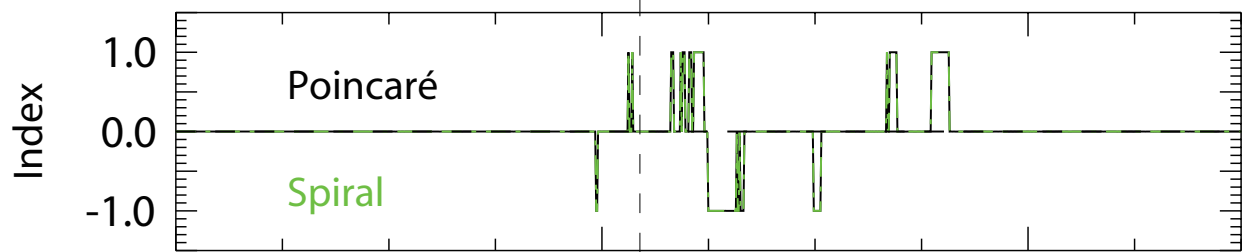

d
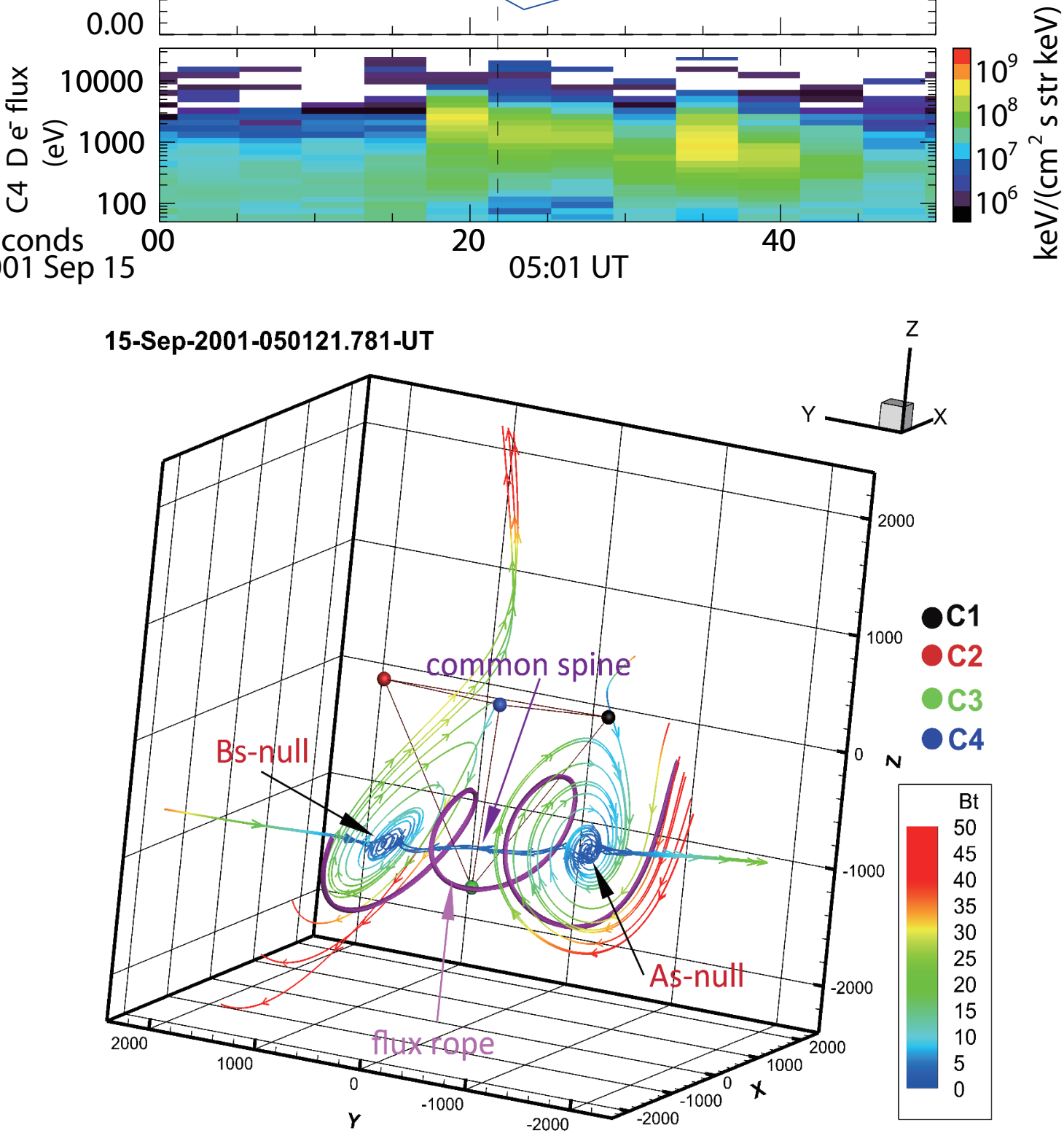